\title{
Extremal Solutions to Periodic Boundary Value Problem of Nabla Integrodifferential Equation of Volterra Type on Time Scales
}

\author{
Yunlong Shi ${ }^{1}$ and Junfang Zhao $^{2}$ \\ ${ }^{1}$ Personnel Office, China University of Geosciences, Beijing 100083, China \\ ${ }^{2}$ School of Science, China University of Geosciences, Beijing 100083, China \\ Correspondence should be addressed to Junfang Zhao; zhao_junfang@163.com
}

Received 12 June 2014; Accepted 24 July 2014; Published 13 October 2014

Academic Editor: Gabriele Bonanno

Copyright (C) 2014 Y. Shi and J. Zhao. This is an open access article distributed under the Creative Commons Attribution License, which permits unrestricted use, distribution, and reproduction in any medium, provided the original work is properly cited.

We firstly establish some new theorems on time scales, and then, by employing them together with a new comparison result and the monotone iterative technique, we show the existence of extremal solutions to the following nabla integrodifferential periodic boundary value problem: $u^{\nabla}(t)=f\left(t, u, \int_{0}^{t} g(t, s) \nabla s\right), t \in[0, a]_{\mathbb{T}}, u(0)=u(\rho(a))$, where $\mathbb{T}$ is a time scale.

\section{Introduction}

In this paper, we are concerned with the existence of extremal solutions to the following nabla integrodifferential periodic boundary value problem:

$$
\begin{gathered}
u^{\nabla}(t)=f\left(t, u, \int_{0}^{t} g(t, s) \nabla s\right), \quad t \in[0, a]_{\mathbb{T}}, \\
u(0)=u(\rho(a)),
\end{gathered}
$$

where $\mathbb{T}$ is a time scale and $f, g$ satisfy

$$
\left(\mathrm{S}_{1}\right) f \in C_{\mathrm{ld}}\left([0, a]_{\mathbb{T}} \times \mathbb{R} \times \mathbb{R}, \mathbb{R}\right), g \in C_{\mathrm{ld}}\left([0, a]_{\mathbb{T}} \times\right.
$$$$
\left.[0, a]_{\mathbb{T}}, \mathbb{R}\right) \text {. }
$$

By proving a new comparison result and developing the monotone iterative technique, we show the extremal solutions of the periodic boundary value problem of nabla integrodifferential equations of Volterra type on time scales.

The study of dynamic equations on time scales has been created in order to unify the study of differential and difference equations. The general idea is to prove a result for a dynamic equation where the domain of the unknown function is a so-called time scale, which may be an arbitrary closed subset of the reals. Many results on this issue have been well documented in the monographs $[1,2]$ written by Bohner and Peterson. Moreover, an integrodifferential equation on time scales (including time scale $\mathbb{R}$ ) finds many applications in various mathematical problems [3]. And this leads to the extensive study of the existence of extremal solutions to such kind of equations; see Agarwal et al. [4], Franco [5], Guo [6], Z. He and X. He [7], Nieto and Rodríguez-López [8], Song [9], $\mathrm{Xu}$ and Nieto [10], Xing et al. [11], and the references therein. However, to the best of the authors' knowledge, most of them are of ordinary integrodifferential equations and delta integrodifferential equations on time scales, while the nabla integrodifferential equations on time scales have rarely been considered up to now; the main reason is that the theory on nabla derivatives on time scales is not complete. So, in order to study PBVP (1) to fill the gap, we need firstly to establish some new theorems on time scales, including the Induction Principle and Mean Value Theorem, which are very important for getting our main results, and this will be shown in Section 2.2.

In addition, monotone iterative technique coupled with the method of upper and lower solutions has been widely used in the treatment of existence results of initial and boundary value problems for nonlinear differential equations in recent years. The basic idea is that using the upper and 
lower solutions as an initial iteration one can construct monotone sequences from a corresponding linear problem, and these sequences converge monotonically to the minimal and maximal solutions of the nonlinear problem. When the method is applied to nabla differential equations on time scales, it needs a suitable nabla differential inequality as a comparison principle; this will be shown in Section 3.

For some other work on time scales, we refer the readers to Aderson [12], Agarwal et al. [13], Tisdell et al. [14, 15], and the references therein.

We will assume the following throughout: by $t \in[0, a]_{\mathbb{T}}$ we mean that $t \in[0, a] \cap \mathbb{T}$, where $0<a$. And we denote $[0, a]_{\mathbb{T}}$ by $J$.

\section{Preliminary}

2.1. Some Definitions and Lemmas. For convenience, in this subsection, we give some definitions and lemmas on time scales, which can be found in book $[1,2]$.

Definition 1 (see [1, page 1]). Let $\mathbb{T}$ be a time scale. For $t \in \mathbb{T}$, one defines the forward jump operator $\sigma: \mathbb{T} \rightarrow \mathbb{T}$ by

$$
\sigma(t):=\inf \{s \in \mathbb{T}: s>t\}
$$

while one defines the backward jump operator $\rho: \mathbb{T} \rightarrow \mathbb{\mathbb { }}$ by

$$
\rho(t):=\sup \{s \in \mathbb{T}: s<t\} .
$$

Definition 2 (see [2, page 47]). If $\mathbb{T}$ has a right-scattered minimum $m$, define $\mathbb{T}_{k}:=\mathbb{T}-m$; otherwise, set $\mathbb{T}_{k}:=\mathbb{T}$. The backward graininess function $v: \mathbb{T}_{k} \rightarrow[0, \infty)$ is defined by

$$
\nu(t):=t-\rho(t) .
$$

Definition 3 (see [1, page 47]). For $f: \mathbb{T} \rightarrow \mathbb{R}$ and $t \in \mathbb{T}_{k}$, define the nabla derivative of $f$ at $t$, denoted by $f^{\nabla}(t)$, to be the number (provided it exists) with the property that, given any $\varepsilon>0$, there is a neighborhood $U$ of $t$ such that

$$
\left|f(\rho(t))-f(s)-f^{\nabla}(t)(\rho(t)-s)\right| \leq \varepsilon|\rho(t)-s|,
$$

for all $s \in U$.

Definition 4 (see [1, page 48]). The function $p$ is $\nu$-regressive if

$$
1-v(t) p(t) \neq 0 \quad \forall t \in \mathbb{T}_{k} .
$$

Define the $\nu$-regressive class of functions on $\mathbb{T}_{k}$ to be

$$
\mathscr{R}_{v}=\{p: \mathbb{T} \rightarrow \mathbb{R}: p \text { is ld-continuous and } \nu \text {-regressive }\} .
$$

If $p, q \in \mathscr{R}_{v}$, then we define circle plus addition by

$$
\left(p \bigoplus_{v} q\right)(t):=p(t)+q(t)-p(t) q(t) v(t) \quad \forall t \in \mathbb{T}_{k} .
$$

Definition 5 (see $[1$, page 48$]$ ). For $p \in \mathscr{R}_{v}$, define circle minus $p$ by

$$
\ominus_{\nu} p:=-\frac{p}{1-p \nu}
$$

Definition 6 (see [1, page 49]). For $h>0$, let

$$
\begin{gathered}
\mathbb{Z}_{h}:=\left\{z \in \mathbb{C},-\frac{\pi}{h}<\operatorname{Im}(z)<\frac{\pi}{h}\right\}, \\
\mathbb{C}_{h}:=\left\{z \in \mathbb{C}, z \neq \frac{1}{h}\right\} .
\end{gathered}
$$

Define the $\nu$-cylinder transformation $\widehat{\xi}_{h}: \mathbb{C}_{h} \rightarrow \mathbb{Z}_{h}$ by

$$
\widehat{\xi}_{h}(z):=-\frac{1}{h} \log (1-z h),
$$

where $\log$ is the principle logarithm function. For $h=0$, we define $\widehat{\xi}_{0}(z)=z$ for all $z \in \mathbb{C}_{0}:=\mathbb{C}$.

Definition 7 (see [1, page 49]). If $p \in \mathscr{R}_{\gamma}$, then we define the nabla exponential function by

$$
\widehat{e}_{p}(t, s):=\exp \left(\int_{s}^{t} \widehat{\xi}_{\nu(\tau)}(p(\tau)) \nabla \tau\right) \quad \forall s, t \in \mathbb{T},
$$

where the $\nu$-cylinder transformation $\widehat{\xi}_{h}$ is as in Definition 6 .

Remark 8. From Definitions 6 and 7 we know that $0 \leq$ $v(t) p(t)<1$ implies $\widehat{e}_{p}(t, s) \geq 1$.

Lemma 9 (see [1, page 51]). Let $p, q \in \mathscr{R}_{v}$ and $s, t, r \in \mathbb{T}$. Then one has the following:

(i) $\widehat{e}_{0}(t, s) \equiv 1$ and $\widehat{e}_{p}(t, t) \equiv 1$;

(ii) $\hat{e}_{p}(\rho(t), s)=(1-v(t) p(t)) \widehat{e}_{p}(t, s)$;

(iii) $1 / \widehat{e}_{p}(t, s)=\widehat{e}_{\ominus_{\nu} p}(t, s)$;

(iv) $\widehat{e}_{p}(t, s)=1 / \widehat{e}_{p}(s, t)=\widehat{e}_{\ominus_{v} p}(s, t)$;

(v) $\widehat{e}_{p}(t, r) \widehat{e}_{p}(r, s)=\widehat{e}_{p}(t, s)$;

(vi) $\widehat{e}_{p}(t, s) \widehat{e}_{q}(t, s)=\widehat{e}_{p \oplus_{v} q}(t, s)$;

(vii) $\widehat{e}_{p}(t, s) / \widehat{e}_{q}(t, s)=\widehat{e}_{p \ominus_{\nu} q}(t, s)$;

(viii) $\left(1 / \widehat{e}_{p}(t, s)\right)^{\nabla}=-p(t) / \widehat{e}_{p}^{\rho}(t, s)$.

Lemma 10 (see $[1$, page 48$]$ ). Assume that $f, g: \mathbb{T} \rightarrow \mathbb{R}$ are nabla differentiable at $t \in \mathbb{T}_{k}$. Then

(i) the sum $f+g: \mathbb{T} \rightarrow \mathbb{R}$ is nabla differentiable at $t$ with

$$
(f+g)^{\nabla}(t)=f^{\nabla}(t)+g^{\nabla}(t)
$$

(ii) the product $f g: \mathbb{T} \rightarrow \mathbb{R}$ is nabla differentiable at $t$, and the product rules

$$
\begin{aligned}
(f g)^{\nabla}(t) & =f^{\nabla}(t) g(t)+f^{\rho}(t) g^{\nabla}(t) \\
& =f(t) g^{\nabla}(t)+f^{\nabla}(t) g^{\rho}(t)
\end{aligned}
$$

(iii) if $g(t) g^{\rho}(t) \neq 0$, then $f / g$ is nabla differentiable at $t$, and we get the quotient rule

$$
\left(\frac{f}{g}\right)^{\nabla}(t)=\frac{f^{\nabla}(t) g(t)+f(t) g^{\nabla}(t)}{g(t) g^{\rho}(t)}
$$

(iv) if $f$ and $f^{\nabla}$ are continuous, then

$$
\left(\int_{a}^{t} f(t, s) \nabla s\right)^{\nabla}=f(\rho(t), t)+\int_{a}^{t} f^{\nabla}(t, s) \nabla s .
$$


2.2. Some New Results on Time Scales. In order to get Theorem 16 which plays an important role in getting our main results, in this subsection, we need firstly to establish Lemmas 11,14 , and 15 . The counterpart about delta derivatives can be found in book $[1,2]$.

Lemma 11 (left-forward induction principle). Let $t_{0} \in \mathbb{T}$ and assume that

$$
\left\{S(t): t \in\left(-\infty, t_{0}\right]\right\}
$$

is a family of statements satisfying the following.

(i) The statement $S\left(t_{0}\right)$ is true.

(ii) If $t \in\left(-\infty, t_{0}\right]$ is left-scattered and $S(t)$ is true, then $S(\rho(t))$ is also true.

(iii) If $t \in\left(-\infty, t_{0}\right]$ is left-dense and $S(t)$ is true, then there is a neighborhood $U$ of $t$ such that $S(s)$ is true for all $s \in U \cap(-\infty, t)$.

(iv) If $t \in\left(-\infty, t_{0}\right)$ is right-dense and $S(s)$ is true for all $s \in\left(t, t_{0}\right]$, then $S(t)$ is true.

Then $S(t)$ is true for all $t \in\left(-\infty, t_{0}\right]$.

Proof. Let

$$
S^{*}:=\left\{t \in\left(-\infty, t_{0}\right]: S(t) \text { is not true }\right\} .
$$

We want to show $S^{*}=\emptyset$. To achieve a contradiction we assume $S^{*} \neq \emptyset$. But since $S^{*}$ is closed and nonempty, we have

$$
\sup S^{*}=: t^{*} \in \mathbb{T} \text {. }
$$

We claim that $S\left(t^{*}\right)$ is true. If $t^{*}=t_{0}$, then $S\left(t^{*}\right)$ is true from (I). If $t^{*} \neq t_{0}$ and $\sigma\left(t^{*}\right)=t^{*}$, then $S\left(t^{*}\right)$ is true from (IV). Finally, if $\sigma\left(t^{*}\right)>t^{*}$, then $S\left(t^{*}\right)$ is true from (II). Hence, in any case,

$$
t^{*} \notin S^{*} \text {. }
$$

Thus, $t^{*}$ cannot be left-scattered (as $t^{*}=\sup S^{*}$ ), and $t^{*} \neq$ $\min \mathbb{\mathbb { }}\left(\right.$ or $\left.S^{*}=\emptyset\right)$. Hence $t^{*}$ is left-dense. But now (III) leads to a contradiction. The proof is complete.

Next, for convenience, we give a definition.

Definition 12. A continuous function $f: \mathbb{T} \rightarrow \mathbb{R}$ is called pre-nabla-differentiable with (region of differentiation) $D$, provided $D \subset \mathbb{T}_{k}, \mathbb{T}_{k} \backslash D$ is countable and contains no leftscattered elements of $\mathbb{T}$, and $f$ is nabla differentiable at each $t \in D$.

Remark 13. This is an example; let $\mathbb{T}:=\mathbb{P}_{2,1}$ and let $f: \mathbb{T} \rightarrow$ $\mathbb{R}$ be defined by

$$
f(t)= \begin{cases}0, & \text { if } t \in \bigcup_{k=0}^{\infty}[3 k, 3 k+1], \\ t-3 k-1, & \text { if } t \in[3 k+1,3 k+2], k \in \mathbb{N}_{0} .\end{cases}
$$

Then $f$ is pre-nabla-differentiable with

$$
D:=\frac{\mathbb{T}}{\bigcup_{k=0}^{\infty}\{3 k+1\}} .
$$

Lemma 14 (Mean Value Theorem). Let $f$ and $g$ be real-valued functions defined on $\mathbb{T}$ and both pre-nabla-differentiable with $D$; then

$$
\left|f^{\nabla}(t)\right| \leq g^{\nabla}(t) \quad \forall t \in D
$$

implies

$$
|f(s)-f(r)| \leq g(s)-g(r) \quad \forall r, s \in \mathbb{T}, r \leq s .
$$

Proof. Let $r, s \in \mathbb{T}$, with $r<s$ and denote $(r, s] \backslash D=\left\{t_{n}: n \in\right.$ $\mathbb{N}$ \}. Let $\varepsilon>0$; we now show by induction that

$$
S(t):|f(s)-f(t)| \leq g(s)-g(t)+\varepsilon\left(s-t+\sum_{t_{n}>t} 2^{-n}\right)
$$

holds for all $t \in[r, s]$. Note that, once we have shown this, the claim of the Mean Value Theorem follows. We now check the conditions given in Lemma 11 as follows.

(I) The statement $S(s)$ is trivially satisfied.

(II) Let $t$ be left-scattered and assume that $S(t)$ holds. Then $t \in D$ and

$$
\begin{aligned}
|f(s)-f(\rho(t))| & \left|f(s)-f(t)+v(t) f^{\nabla}(t)\right| \\
\leq & |f(s)-f(t)|+v(t) f^{\nabla}(t) \\
\leq & g(s)-g(t)+\varepsilon\left(s-t+\sum_{t_{n}>t} 2^{-n}\right)+v(t) g^{\nabla}(t) \\
= & g(s)-g(t)+\varepsilon\left(s-t+\sum_{t_{n}>t} 2^{-n}\right) \\
& +g(t)-g(\rho(t)) \\
= & g(s)-g(\rho(t))+\varepsilon\left(s-t+\sum_{t_{n}>t} 2^{-n}\right) \\
& <g(s)-g(\rho(t))+\varepsilon\left(s-t+\sum_{t_{n}>\rho(t)} 2^{-n}\right) .
\end{aligned}
$$

Therefore $S(\rho(t))$ is true.

(III) Suppose $S(t)$ is true and $t \neq s$ is left-dense; that is, $\rho(t)=t$. We consider two cases; namely, $t \in D$ and $t \notin D$. First of all, suppose $t \in D$. Then $f$ and $g$ are differentiable at $t$ and hence there exists a neighborhood of $t$ with

$$
\begin{array}{ll}
\left|f(t)-f(\tau)-f^{\nabla}(\tau)(t-\tau)\right| \leq \frac{\varepsilon}{2}|t-\tau| & \forall \tau \in U, \\
\left|g(t)-g(\tau)-g^{\nabla}(\tau)(t-\tau)\right| \leq \frac{\varepsilon}{2}|t-\tau| & \forall \tau \in U .
\end{array}
$$

Thus

$$
\begin{gathered}
|f(t)-f(\tau)| \leq\left[\left|f^{\nabla}(t)\right|+\frac{\varepsilon}{2}\right]|t-\tau| \quad \forall \tau \in U, \\
g(t)-g(\tau)-g^{\nabla}(t)(t-\tau) \geq-\frac{\varepsilon}{2}|t-\tau| \quad \forall \tau \in U .
\end{gathered}
$$


That is,

$$
g^{\nabla}(t)(t-\tau) \leq g(t)-g(\tau)+\frac{\varepsilon}{2}|t-\tau| \quad \forall \tau \in U
$$

Hence we have for all $\tau \in U \cap(-\infty, t)$

$$
\begin{aligned}
|f(s)-f(\tau)| \leq & |f(s)-f(t)|+|f(t)-f(\tau)| \\
\leq & g(s)-g(t)+\varepsilon\left(s-t+\sum_{t_{n}>t} 2^{-n}\right) \\
& +\left[\left|f^{\nabla}(t)\right|+\frac{\varepsilon}{2}\right]|t-\tau| \\
\leq & g(s)-g(t)+\varepsilon\left(s-t+\sum_{t_{n}>t} 2^{-n}\right) \\
& +\left[g^{\nabla}(t)+\frac{\varepsilon}{2}\right]|t-\tau| \\
\leq & g(s)-g(t)+\varepsilon(s-t)+\varepsilon \sum_{t_{n}>t} 2^{-n} \\
& +\left[g(t)+\frac{\varepsilon}{2}\right]|t-\tau| \\
\leq & g(s)-g(t)+\varepsilon(s-t)+\varepsilon \sum_{t_{n}>t} 2^{-n} \\
= & g(s)-g(\tau)+\varepsilon\left(s-\tau+\sum_{t_{n}>\tau} 2^{-n}\right) \\
& +g(t)-g(\tau)+\frac{\varepsilon}{2}(t-\tau)+\frac{\varepsilon}{2}(t-\tau) \\
& g(\tau)+\varepsilon\left(s-\tau+\sum_{t_{n}>t} 2^{-n}\right) \\
& \\
& \\
&
\end{aligned}
$$

Thus $S(\tau)$ is true for all $\tau \in U \cap(-\infty, t)$.

For the second case, suppose $t \notin D$. Then $t=t_{m}$ for $m \in \mathbb{N}$. Since $f$ and $g$ are pre-nabla-differentiable, they are continuous and hence there exists a neighborhood $U$ of $t$ with

$$
\begin{aligned}
& |f(\tau)-f(t)| \leq \frac{\varepsilon}{2} 2^{-m} \quad \forall \tau \in U, \\
& |g(\tau)-g(t)| \leq \frac{\varepsilon}{2} 2^{-m} \quad \forall \tau \in U .
\end{aligned}
$$

Therefore

$$
g(t)-g(\tau) \geq-\frac{\varepsilon}{2} 2^{-m} \quad \forall \tau \in U
$$

That is,

$$
-g(t) \leq \frac{\varepsilon}{2} 2^{-m}-g(\tau) \quad \forall \tau \in U
$$

and hence

$$
\begin{aligned}
\mid f(s) & -f(\tau) \mid \\
& \leq|f(s)-f(t)|+|f(t)-f(\tau)| \\
& \leq g(s)-g(t)+\varepsilon\left(s-t+\sum_{t_{n}>t} 2^{-n}\right)+\frac{\varepsilon}{2} 2^{-m} \\
& \leq g(s)-g(\tau)+\frac{\varepsilon}{2} 2^{-m}+\varepsilon\left(s-t+\sum_{t_{n}>t} 2^{-n}\right)+\frac{\varepsilon}{2} 2^{-m} \\
& =g(s)-g(\tau)+\varepsilon\left(s-t+\sum_{t_{n}>t} 2^{-n}\right)+\varepsilon 2^{-m} \\
& \leq g(s)-g(\tau)+\varepsilon\left(s-t+\sum_{t_{n}>\tau} 2^{-n}\right) .
\end{aligned}
$$

Thus again $S(\tau)$ follows for all $\tau \in U \cap(-\infty, t)$.

(IV) Now let $t$ be right-dense and suppose that $S(\tau)$ is true for $\tau>t$. Then

$$
\begin{aligned}
\lim _{\tau \rightarrow t^{+}}|f(s)-f(\tau)| \\
\leq \lim _{\tau \rightarrow t^{+}}\left\{g(s)-g(\tau)+\varepsilon\left(s-\tau+\sum_{t_{n}>\tau} 2^{-n}\right)\right\} \\
\leq \lim _{\tau \rightarrow t^{+}}\left\{g(s)-g(\tau)+\varepsilon\left(s-\tau+\sum_{t_{n}>t} 2^{-n}\right)\right\} \\
\quad \leq g(s)-g(\tau)+\varepsilon\left(s-t+\sum_{t_{n}>t} 2^{-n}\right)
\end{aligned}
$$

implies that $S(t)$ is true as both $f$ and $g$ are continuous at $t$.

Lemma 15. Suppose $f$ is pre-nabla-differentiable with $D$ and $U$ is a compact interval with endpoints $r, s \in \mathbb{T}$; then

$$
|f(s)-f(r)| \leq\left\{\sup _{t \in U_{k} \cap D}\left|f^{\nabla}\right|\right\}|s-r|, \quad \text { for } t \in \mathbb{T} .
$$

Proof. Suppose $f$ is pre-nabla-differentiable with $D$ and $r, s \in$ $\mathbb{}$ with $r \leq s$. Defining

$$
g(t)=\left\{\sup _{\tau \in[r, s]_{k}}\left|f^{\nabla}(\tau)\right|\right\}(t-r), \quad \forall t \in D \cap[r, s]_{k},
$$

then

$$
g^{\nabla}(t)=\left\{\sup _{\tau \in[r, s]_{k}}\left|f^{\nabla}(\tau)\right|\right\} \geq\left|f^{\nabla}(t)\right| .
$$

By Lemma 14, we get

$$
g(s)-g(t) \geq|f(s)-f(t)| \quad \forall t \in[r, s]
$$


so that

$$
\begin{aligned}
|f(s)-f(r)| & \leq g(s)-g(r)=g(s) \\
& =\left\{\sup _{\tau \in[r, s]_{k}}\left|f^{\nabla}(\tau)\right|\right\}(s-r) .
\end{aligned}
$$

This completes the proof.

Theorem 16. Suppose $f_{n}: \mathbb{T} \rightarrow \mathbb{X}$ is pre-nabla-differentiable with $D$ for each $n \in \mathbb{N}$. Assume that for each $t \in \mathbb{T}_{k}$ there exists a compact interval neighborhood $U(t)$ such that the sequence

$$
\left\{f_{n}^{\nabla}\right\}_{n \in \mathbb{N}} \text { converges uniformly on } U(t) \cap D .
$$

Then the limit mapping $f=\lim _{n \rightarrow \infty} f_{n}$ is predifferentiable with $D$ and one has

$$
f^{\nabla}(t)=\lim _{n \rightarrow \infty} f_{n}^{\nabla}(t) \quad \forall t \in D .
$$

Proof. Let $t \in D$. Without loss of generality we can assume that $\rho(t) \in U(t)$. Letting $\varepsilon>0, t \in \mathbb{T}_{k}$, there exists $N \in \mathbb{N}$ such that

$$
\sup _{s \in U(t) \cap D}\left|\left(f_{n}-f_{m}\right)^{\nabla}(s)\right| \text { is finite } \forall m, n \geq N .
$$

Also by Lemma 15

$$
\begin{aligned}
\mid\left(f_{n}-\right. & \left.f_{m}\right)(r)-\left(f_{n}-f_{m}\right)(\rho(t)) \mid \\
& \leq\left\{\sup _{s \in U(t) \cap D}\left|\left(f_{n}-f_{m}\right)^{\nabla}(s)\right|\right\}|r-\rho(t)|
\end{aligned}
$$

holds for all $r \in U(t)$, and $m, n>N$. Since $\left\{f_{n}^{\nabla}\right\}_{n \in \mathbb{N}}$ converges uniformly on $U(t) \cap D$, there exists $\widetilde{N}>N$ such that

$$
\sup _{s \in U(t) \cap D}\left|\left(f_{n}-f_{m}\right)^{\nabla}(s)\right| \leq \frac{\varepsilon}{3} \quad \forall m, n>\widetilde{N} .
$$

Hence,

$$
\left|\left(f_{n}-f_{m}\right)(r)-\left(f_{n}-f_{m}\right)(\rho(t))\right| \leq \frac{\varepsilon}{3}|r-\rho(t)|
$$

for all $r \in U(t)$, and $m, n>N$ so that, by letting $m \rightarrow \infty$,

$$
\left|\left(f_{n}-f\right)(r)-\left(f_{n}-f\right)(\rho(t))\right| \leq \frac{\varepsilon}{3}|r-\rho(t)|,
$$

for all $r \in U(t)$, and $n>\widetilde{N}$. Let

$$
g=\lim _{n \rightarrow \infty} f_{n}^{\nabla} .
$$

Then there exists $\widetilde{m}>\widetilde{N}$ such that

$$
\left|f_{\widetilde{m}}^{\nabla}(\rho(t))-g(\rho(t))\right| \leq \frac{\varepsilon}{3},
$$

and since $f_{\widetilde{m}}$ is nabla differentiable at $t$, there also exists a neighborhood $W$ of $t$ with

$$
\begin{aligned}
& \left|f_{\widetilde{m}}(\rho(t))-f_{\widetilde{m}}(r)-f_{\widetilde{m}}^{\nabla}(\rho(t))(\rho(t)-r)\right| \\
& \quad \leq \frac{\varepsilon}{3}|\rho(t)-r| \quad \forall r \in W .
\end{aligned}
$$

Altogether we have now, for all $r \in U(t) \cap W$,

$$
\begin{aligned}
\mid f(\rho(t))- & f(r)-g(t)(\rho(t)-r) \mid \\
=\mid & f(\rho(t))-f_{\widetilde{m}}(\rho(t))+f_{\widetilde{m}}(\rho(t)) \\
& +f_{\widetilde{m}}(r)-f_{\widetilde{m}}(r)-f(r)+f_{\widetilde{m}}^{\nabla}(t)(\rho(t)-r) \\
& \quad-f_{\widetilde{m}}^{\nabla}(t)(\rho(t)-r)-g(t)(\rho(t)-r) \mid \\
\leq & \left|\left(f_{\widetilde{m}}-f\right)(\rho(t))-\left(f_{\widetilde{m}}-f\right)(r)\right| \\
& +\left|f_{\widetilde{m}}(\rho(t))-f_{\widetilde{m}}(r)-f_{\widetilde{m}}^{\nabla}(t)(\rho(t)-r)\right| \\
& +\left|\left[f_{\widetilde{m}}^{\nabla}-g(t)\right](\rho(t)-r)\right| \\
\leq & \frac{\varepsilon}{3}|\rho(t)-r|+\frac{\varepsilon}{3}|\rho(t)-r|+\frac{\varepsilon}{3}|\rho(t)-r| \\
= & \varepsilon|\rho(t)-r|,
\end{aligned}
$$

which implies that $f$ is nabla differentiable at $t$ with $f^{\nabla}(t)=$ $g(t)$; that is, $f^{\nabla}(t)=\lim _{n \rightarrow \infty} f_{n}^{\nabla}$; the proof is complete.

\section{Some Important Lemmas}

In this section, we will give some lemmas which are important for the main results.

Lemma 17 (comparison result). Suppose that there is a function $g(t, s) \in C_{l d}\left[J \times J, \mathbb{R}^{+}\right]$and $G=\max \{g(t, s)$ : $t, s \in J \times J\}>0$. Assume that there exist a positive function $p(t) \in \mathscr{R}_{\nu}$ and a nonnegative function $q(t) \in \mathscr{R}_{\nu}$ on $J$, such that $\alpha=\sup _{t \in J}\{v(t) p(t)\}<1$ and

$$
\begin{gathered}
u^{\nabla}(t) \leq-p(t) u(t)-q(t) \int_{0}^{t} g(t, s) u(s) \nabla s, \quad t \in[0, a]_{\mathbb{T}}, \\
u(0) \leq u(\rho(a)) .
\end{gathered}
$$

Then $u(t) \leq 0$ for all $t \in J$ provided that $Q G M \rho(a) / p_{0} \leq$ 1 , where $Q=\max _{t \in J}\{q(t)\}, p_{0}=\min _{t \in J}\{p(t)\}, M=$ $\left\{\widehat{e}_{\Theta_{\nu}(-p)}(\rho(a), 0)-1\right\}$.

Proof. Denote $m(t)=\widehat{e}_{\Theta_{v}(-p)}(t, 0) u(t)$. By Definition 5 and (52), we know that $\ominus_{v}(-p)=p(t) /(1+p v)$; thus, by Lemma 9, we have

$$
\begin{aligned}
m^{\nabla}(t)= & \Theta_{\nu}(-p) \widehat{e}_{\Theta_{\nu}(-p)}(t, 0) u(t)+\widehat{e}_{\Theta_{\nu}(-p)}(\rho(t), 0) u^{\nabla}(t) \\
\leq & \frac{p(t)}{1+p \nu} \widehat{e}_{\Theta_{\nu}(-p)}(t, 0) u(t) \\
& +\left(1-v \Theta_{\nu}(-p)\right) \widehat{e}_{\theta_{\nu}(-p)}(t, 0) \\
& \times\left(-p(t) u(t)-q(t) \int_{0}^{t} g(t, s) u(s) \nabla s\right) \\
= & \frac{p(t)}{1+p \nu} \widehat{e}_{\Theta_{\nu}(-p)}(t, 0) u(t)-\frac{p(t)}{1+p \nu} \widehat{e}_{\Theta_{\nu}(-p)}(t, 0) u(t)
\end{aligned}
$$




$$
\begin{aligned}
& -\frac{q(t)}{1+p \nu} \widehat{e}_{\Theta_{\nu}(-p)}(t, 0) \int_{0}^{t} g(t, s) u(s) \nabla s \\
= & -\frac{q(t)}{1+p v} \widehat{e}_{\ominus_{\nu}(-p)}(t, 0) \int_{0}^{t} g(t, s) u(s) \nabla s \\
= & -\frac{q(t)}{1+p \nu} \widehat{e}_{\ominus_{\nu}(-p)}(t, 0) \int_{0}^{t} g(t, s) m(s) \hat{e}_{\ominus_{\nu}(-p)}(0, s) \nabla s \\
= & -\frac{q(t)}{1+p v} \int_{0}^{t} g(t, s) m(s) \hat{e}_{\Theta_{\nu}(-p)}(t, s) \nabla s .
\end{aligned}
$$

Next, we try to show that $m(t) \leq 0$ for all $t \in J$. Otherwise, we have one of the following two cases:

(i) $m(t) \geq 0$ for all $t \in J$ and $\sup _{t \in J}\{m(t)\}>0$;

(ii) there exists $t_{1}, t_{2} \in J$ such that $p\left(t_{1}\right)>0$ and $p\left(t_{2}\right)<$ 0 .

In case (i), since $\nu(t) p(t) \geq 0$ and $\alpha=\sup \{v(t) p(t)\}<$ 1 , we have $\ominus_{\gamma}(-p)=p(t) /(1+\nu(t) p(t)) \geq 0$; also $0<1-$ $\nu(t) \ominus_{\gamma}(-p)=1-\nu(p /(1+\nu(t) p(t)))=(1 /(1+\nu(t) p(t)))<1$, so, by Remark 8 , we have $\widehat{e}_{\ominus_{\nu}(-p)}(t, 0) \geq 1, t \in J$. Together with

$$
u(\rho(a)) \geq u(0)=\widehat{e}_{-p}(0,0) m(0)=m(0) \geq 0,
$$

it follows that $m(\rho(a))=\widehat{e}_{\Theta_{\nu}(-p)}(\rho(a), 0) u(\rho(a)) \geq u(\rho(a)) \geq$ $m(0)$.

On the other hand, from (53), we have

$$
m^{\nabla}(t) \leq-\frac{q(t)}{1+\nu p} \int_{0}^{t} g(t, s) m(s) \widehat{e}_{\ominus_{\nu}(-p)}(t, s) \nabla s \leq 0,
$$

which implies that $m(t)$ is nonincreasing and hence $m(0) \geq$ $m(\rho(a))$. Thus, we have $m(t)=c=$ const. $>0$. Therefore, from $m(t)=\widehat{e}_{\Theta_{\gamma}(-p)}(t, 0) u(t)$, we have $u(t)=c \widehat{e}_{-p}(t, 0)$ for all $t \in J$. Since $\widehat{e}_{-p}(t, 0)$ is positive and decreasing on $J, u(0)>$ $u(\rho(a))$ holds. This is a contradiction.

In case (ii), we have two subcases:

$$
\left(\mathrm{ii}_{1}\right): m(\rho(a)) \geq 0, \quad\left(\mathrm{ii}_{2}\right): m(\rho(a))<0 .
$$

When ( $\left.\mathrm{ii}_{1}\right)$ holds, suppose that $m\left(t_{2}\right)=-\lambda=: \min _{t \in J} m(t)$ with $\lambda>0$, and we claim that there exists $t_{3} \in\left[t_{2}, \rho(a)\right)$ such that $m^{\nabla}\left(t_{3}\right) \geq \lambda / \rho(a)$. Otherwise, $m^{\nabla}(t)<\lambda / \rho(a)$ for all $t \in$ $\left[t_{2}, \rho(a)\right)$; it follows that

$$
m(\rho(a))=m\left(t_{2}\right)+\int_{t_{2}}^{\rho(a)} m^{\nabla}(s) \nabla s<-\lambda+\frac{\lambda}{\rho(a)} \rho(a)=0,
$$

which is a contradiction. Further,

$$
\begin{aligned}
& m^{\nabla}\left(t_{3}\right) \\
& \quad \leq-\frac{q\left(t_{3}\right)}{1+v p} \int_{0}^{t_{3}} g k\left(t_{3}, s\right) m(s) \widehat{e}_{\ominus_{\nu}(-p)}\left(t_{3}, s\right) \nabla s \\
& \quad=\frac{q\left(t_{3}\right)}{1+v p} \int_{0}^{t_{3}} g\left(t_{3}, s\right)[-m(s)] \widehat{e}_{\ominus_{\nu}(-p)}\left(t_{3}, s\right) \nabla s
\end{aligned}
$$

$$
\begin{aligned}
& \leq Q G \lambda \int_{0}^{t_{3}} \widehat{e}_{\Theta_{\nu}(-p)}\left(t_{3}, s\right) \nabla s, \\
& \int_{0}^{t_{3}} \widehat{e}_{\Theta_{\nu}(-p)}\left(t_{3}, s\right) \nabla s \\
&=\widehat{e}_{\ominus_{\nu}(-p)}\left(t_{3}, 0\right) \int_{0}^{t_{3}} \widehat{e}_{-p}(t, 0) \nabla s \\
&=\left.\frac{1}{p} \widehat{e}_{\Theta_{\nu}(-p)}\left(t_{3}, 0\right)\left[-\widehat{e}_{-p}(s, 0)\right]\right|_{t=0} ^{t=t_{3}} \\
& \leq \frac{1}{p_{0}} \widehat{e}_{\Theta_{\nu}(-p)}\left(t_{3}, 0\right)\left[1-\widehat{e}_{-p}\left(t_{3}, 0\right)\right] \\
&=\frac{1}{p_{0}}\left[\widehat{e}_{\Theta_{\nu}(-p)}\left(t_{3}, 0\right)-1\right] \\
&<\frac{1}{p_{0}}\left[\widehat{e}_{\Theta_{v}(-p)}(\rho(a), 0)-1\right] .
\end{aligned}
$$

Thus, $m^{\nabla}\left(t_{3}\right)<Q G M \lambda / p_{0}$. Then it follows, together with $m^{\nabla}\left(t_{3}\right) \geq \lambda / \rho(a)$, that $Q G M \rho(a) / p_{0}>1$, provided that $Q G M \rho(a) / p_{0} \leq 1$. It is a contradiction and therefore ( $\mathrm{ii}_{1}$ ) cannot occur.

When $\left(\mathrm{ii}_{2}\right)$ holds, we have $\widehat{e}_{-p}(t, 0)>0$. Thus

$$
\begin{aligned}
m(0) & =\widehat{e}_{\Theta_{\nu}(-p)}(0,0) u(0) \leq u(\rho(a)) \\
& =\widehat{e}_{-p}(\rho(a), 0) m(\rho(a))<0 .
\end{aligned}
$$

Let $t_{4}=\inf \{t: m(t)>0, t \in J\}$, and choose $t_{5}$ such that $m\left(t_{5}\right)=\min \left\{m(t), t \in\left[0, t_{4}\right]\right\}=: \lambda$ with $\lambda>0$. As in (ii $\left.{ }_{1}\right)$, we can similarly prove that there exists a $t_{6} \in\left[t_{5}, t_{4}\right)$ such that $m^{\nabla}\left(t_{6}\right) \geq \lambda / \rho(a)$. On the other hand, deducing as before, we have

$$
\begin{aligned}
m^{\nabla}\left(t_{6}\right) & \leq \frac{-q\left(t_{6}\right)}{1+\nu p} \int_{0}^{t_{6}} g\left(t_{6}, s\right) m(s) \widehat{e}_{\ominus_{\nu}(-p)}\left(t_{6}, s\right) \nabla s \\
& <\frac{Q G M \lambda}{p_{0}} .
\end{aligned}
$$

Thus it follows that $Q G M \rho(a) / p_{0}>1$, which contradicts the condition $Q G M \rho(a) / p_{0} \leq 1$.

To sum up, we have $m(t) \leq 0$ for all $t \in J$. Thus, $u(t) \leq 0$ for all $t \in J$. The proof is completed.

Lemma 18 (comparison result). Suppose that $g(t, s), p(t)$, and $q(t)$ satisfy all the conditions in Lemma 17 and $u$ satisfy (52); then $u(t) \leq 0$ for all $t \in J$ provided that $\rho(a)[P+$ $\rho(a) Q G] \leq 1$, where $Q, G$ are defined as in Lemma 17, and $P=\max _{t \in J}\{p(t)\}$.

Proof. If the conclusion is not true, we have one of the following two cases:

(i) $u(t) \geq 0$ for all $t \in J$ and $\sup _{t \in J}\{u(t)\}>0$;

(ii) there exist $t_{1}, t_{2} \in J$ such that $u\left(t_{1}\right)>0$ and $u\left(t_{2}\right)<0$. 
In case (i), we have

$$
u^{\nabla}(t) \leq-p(t) u(t)-q(t) \int_{0}^{t} g(t, s) u(s) \nabla s \leq 0,
$$

which implies that $u(t)$ is nonincreasing and so $u(0) \geq$ $u(\rho(a))$. Then it follows from $u(0) \leq u(\rho(a))$ that $u(t)=c$, where $c$ is a constant. Hence $u^{\nabla}(t) \equiv 0$. On the other hand,

$$
\begin{aligned}
u^{\nabla}(t) & \leq-p(t) u(t)-q(t) \int_{0}^{t} g(t, s) u(s) \nabla s \\
& =-p(t) c-q(t) \int_{0}^{t} g(t, s) c \nabla s \\
& \leq-p_{0} c<0,
\end{aligned}
$$

where $p_{0}=\min _{t \in J}\{p(t)\}$. This is a contradiction.

In case (ii), we have two subcases:

$$
\left(\mathrm{ii}_{1}\right): u(\rho(a)) \geq 0, \quad\left(\mathrm{ii}_{2}\right): u(\rho(a))<0 .
$$

When ( $\mathrm{ii}_{1}$ ) holds, suppose that $u\left(t_{3}\right)=-\lambda=: \min _{t \in J}\{u(t)\}$ with $\lambda>0$, and we claim that there exists a $t_{4} \in\left[t_{3}, \rho(a)\right)$ such that $u^{\nabla}\left(t_{4}\right) \geq \lambda / \rho(a)$. Otherwise $u^{\nabla}(t)<\lambda / \rho(a)$ for all $t \in\left[t_{3}, \rho(a)\right)$; it therefore follows that

$$
u(\rho(a))=u\left(t_{3}\right)+\int_{t_{3}}^{\rho(a)} u^{\nabla}(s) \nabla s<-\lambda+\frac{\lambda}{\rho(a)} \rho(a),
$$

which is a contradiction. On the other hand,

$$
\begin{aligned}
u^{\nabla}\left(t_{4}\right) & \leq-p\left(t_{4}\right) u\left(t_{4}\right)-q\left(t_{4}\right) \int_{0}^{t_{4}} g\left(t_{4}, s\right) u(s) \nabla s \\
& \leq \lambda P+\lambda Q G t_{4}<\lambda P+\lambda Q G \rho(a) .
\end{aligned}
$$

Then, together with $u^{\nabla}\left(t_{4}\right) \geq \lambda / \rho(a)$, it follows that

$$
\rho(a)[P+\rho(a) Q G]>1 .
$$

It is a contradiction and therefore ( $\mathrm{ii}_{1}$ ) does not hold. Similarly, we can prove that case $\left(\mathrm{ii}_{2}\right)$ is also wrong. Hence the proof is complete.

Lemma 19 (existence result). Assume that all the assumptions on $g(t, s), q(t)$, and $p(t)$ in Lemma 17 are satisfied. Then, for any $h(t) \in C_{1 \mathrm{~d}}[J, \mathbb{R}]$, the periodic boundary value problem

$$
\begin{gathered}
u^{\nabla}(t)=-p(t) u(t)-q(t)(G u)(t)+h(t), \\
u(0)=u(\rho(a))
\end{gathered}
$$

has a unique solution $u_{h}(t)$ provided $\rho(a) Q G<p_{0}$, where

$$
(G u)(t)=\int_{0}^{t} g(t, s) u(s) \nabla s, \quad p_{0}=\min _{t \in J}\{p(t)\}>0 .
$$

Proof. We will prove the conclusion by Banach contraction Principle. First, define a Banach Space as follows:

$$
E=C_{\mathrm{ld}}[J, \mathbb{R}] \text { be endowed with the norm }
$$

$$
\|u\|=\max _{t \in J}\{u(t)\} .
$$

We define an operator on $E$ as

$(T u)(t)$

$$
\begin{aligned}
& =\widehat{e}_{-p}(t, 0) \\
& \times\left(\frac{1}{\widehat{e}_{\Theta_{\nu}(-p)}(\rho(a), 0)-1}\right. \\
& \quad \times \int_{0}^{\rho(a)} \hat{e}_{-p}(0, \rho(s))[h(s)-q(s)(G u)(s)] \nabla s \\
& \left.\quad+\int_{0}^{t} \widehat{e}_{-p}(0, \rho(s))[h(s)-q(s)(G u)(s)] \nabla s\right), \quad t \in J .
\end{aligned}
$$

For any two functions $u_{1}, u_{2} \in E$, there holds

$$
\begin{aligned}
& \left|\left(T u_{1}\right)(t)-\left(T u_{2}\right)(t)\right| \\
& =\mid \frac{\widehat{e}_{-p}(t, 0)}{\widehat{e}_{\Theta_{\nu}}(-p)}(\rho(a), 0)-1 \\
& \quad \times \int_{0}^{\rho(a)} \widehat{e}_{-p}(0, \rho(s)) q(s)\left[\int_{0}^{s} g(t, \tau)\left(u_{2}-u_{1}\right)(\tau) \nabla \tau\right] \nabla s \\
& \quad+\widehat{e}_{-p}(t, 0) \\
& \quad \times \int_{0}^{t} \widehat{e}_{-p}(0, \rho(s)) q(s)\left[\int_{0}^{s} g(t, \tau)\left(u_{2}-u_{1}\right)(\tau) \nabla \tau\right] \nabla s \mid,
\end{aligned}
$$

where the integral

$$
\begin{aligned}
& 0 \leq \int_{0}^{t} \widehat{e}_{-p}(0, \rho(s)) \nabla s=\int_{0}^{t} \widehat{e}_{\Theta_{\nu}(-p)}(\rho(s), 0) \nabla s \\
& =\int_{0}^{t}\left(1-\nu \ominus_{\nu}(-p)\right) \widehat{e}_{\Theta_{\nu}(-p)}(s, 0) \nabla s \\
& =\int_{0}^{t}\left(1-v \frac{p}{1+\nu p}\right) \widehat{e}_{\ominus_{\nu}(-p)}(s, 0) \nabla s \\
& =\int_{0}^{t} \frac{1}{1+\nu p} \widehat{e}_{\ominus_{\nu}(-p)}(s, 0) \nabla s \\
& =\int_{0}^{t} \frac{1}{p(s)} \cdot\left(\ominus_{\nu}(-p)\right) \widehat{e}_{\ominus_{\nu}(-p)}(s, 0) \nabla s \\
& \leq \frac{1}{p_{0}} \int_{0}^{t}\left(\ominus_{\nu}(-p)\right) \widehat{e}_{\ominus_{\nu}(-p)}(s, 0) \nabla s \\
& =\frac{1}{p_{0}}\left[\widehat{e}_{\Theta_{\nu}(-p)}(t, 0)-\widehat{e}_{\Theta_{\nu}(-p)}(0,0)\right] \\
& =\frac{1}{p_{0}}\left(\widehat{e}_{\Theta_{\nu}(-p)}(t, 0)-1\right) .
\end{aligned}
$$


Thus we have

$$
\begin{aligned}
& \left\|\left(T u_{1}\right)(t)-\left(T u_{2}\right)(t)\right\| \\
& \leq \frac{\widehat{e}_{-p}(t, 0)}{\widehat{e}_{\ominus_{\nu}(-p)}(\rho(a), 0)-1}\left(\widehat{e}_{\Theta_{\nu}(-p)}(\rho(a), 0)-1\right) \\
& \quad \times \frac{\rho(a) Q G}{p_{0}}\left\|u_{1}-u_{2}\right\| \\
& \quad+\widehat{e}_{-p}(t, 0)\left(\widehat{e}_{\Theta_{\nu}(-p)}(t, 0)-1\right) \frac{\rho(a) Q G}{p_{0}}\left\|u_{1}-u_{2}\right\| \\
& =\left(\widehat{e}_{-p}(t, 0)+1-\widehat{e}_{-p}(t, 0)\right) \frac{\rho(a) Q G}{p_{0}}\left\|u_{1}-u_{2}\right\| \\
& =\frac{\rho(a) Q G}{p_{0}}\left\|u_{1}-u_{2}\right\| .
\end{aligned}
$$

This implies by condition $\rho(a) Q G<p_{0}$ that $T$ is a contraction operator on $E$ and therefore by Banach Contraction Principle there exists exactly one $u(t) \in E$ such that $u=T u$; that is,

$u(t)$

$$
\begin{aligned}
& =\widehat{e}_{-p}(t, 0) \\
& \times\left[\frac{1}{\widehat{e}_{\Theta_{\nu}(-p)}(\rho(a), 0)-1}\right. \\
& \quad \times \int_{0}^{\rho(a)} \widehat{e}_{-p}(0, \rho(s))[h(s)-q(s)(G u)(s)] \nabla s \\
& \left.\quad+\int_{0}^{t} \widehat{e}_{-p}(0, \rho(s))[h(s)-q(s)(G u)(s)] \nabla s\right], \quad t \in J .
\end{aligned}
$$

Next, we will show that $u(t)$ is a solution of (67). In fact,

$$
\begin{aligned}
u^{\nabla}(t)= & (-p) \widehat{e}_{-p}(t, 0) \frac{1}{\widehat{e}_{\Theta_{\nu}(-p)}(\rho(a), 0)-1} \\
& \times \int_{0}^{\rho(a)} \widehat{e}_{-p}(0, \rho(s))[h(s)-q(s)(G u)(s)] \nabla s \\
& +(-p) \widehat{e}_{-p}(t, 0) \\
& \times \int_{0}^{t} \widehat{e}_{-p}(0, \rho(s))[h(s)-q(s)(G u)(s)] \nabla s \\
& +\widehat{e}_{-p}(\rho(t), 0) \widehat{e}_{-p}(0, \rho(t))[h(t)-q(t)(G u)(t)] \\
= & (-p) \widehat{e}_{-p}(t, 0) \\
& \times\left(\frac{1}{\hat{e}_{\ominus_{\nu}}(-p)}(\rho(a), 0)-1\right. \\
& \times \int_{0}^{\rho(a)} \widehat{e}_{-p}(0, \rho(s))[h(s)-q(s)(G u)(s)] \nabla s \\
& \left.+\int_{0}^{t} \widehat{e}_{-p}(0, \rho(s))[h(s)-q(s)(G u)(s)] \nabla s\right)
\end{aligned}
$$

$$
\begin{aligned}
& -q(t)(G u)(t)+h(t) \\
= & -p u(t)-q(t)(G u)(t)+h(t) .
\end{aligned}
$$

Moreover, by Lemma 9(iii), there holds

$$
\begin{aligned}
& u(\rho(a)) \\
& =\widehat{e}_{-p}(\rho(a), 0) \\
& \times\left[\frac{1}{\widehat{e}_{\ominus_{\gamma}(-p)}(\rho(a), 0)-1}\right. \\
& \times \int_{0}^{\rho(a)} \widehat{e}_{-p}(0, \rho(s))[h(s)-q(s)(G u)(s)] \nabla s \\
& \left.+\int_{0}^{\rho(a)} \widehat{e}_{-p}(0, \rho(s))[h(s)-q(s)(G u)(s)] \nabla s\right] \\
& =\widehat{e}_{-p}(\rho(a), 0)\left[\frac{1}{\widehat{e}_{\ominus_{\nu}(-p)}(\rho(a), 0)-1}+1\right] \\
& \times \int_{0}^{\rho(a)} \widehat{e}_{-p}(0, \rho(s))[h(s)-q(s)(G u)(s)] \nabla s \\
& =\frac{1}{\widehat{e}_{\ominus_{\nu}(-p)}(\rho(a), 0)-1} \\
& \times \int_{0}^{\rho(a)} \widehat{e}_{-p}(0, \rho(s))[h(s)-q(s)(G u)(s)] \nabla s \\
& =u(0) \text {. }
\end{aligned}
$$

Thus, the proof is complete.

Lemma 20 (compact result). Assume that $\left\{f_{n}\right\}_{n \in N}$ is a function sequence on J satisfying the following conditions:

(i) $\left\{f_{n}\right\}_{n \in N}$ is bounded on $J$;

(ii) $\left\{f_{n}^{\nabla}\right\}_{n \in N}$ is bounded on J.

Then there is a subsequence of $\left\{f_{n}\right\}_{n \in N}$ that converges uniformly on $J$.

Proof. From the assumption, there exists a positive number $R$ such that $\left|f_{n}^{\nabla}\right| \leq R$ for all $t \in J$ and $n \in \mathbb{N}$. Since $J$ is bounded, we can choose $\Omega=\left\{r_{1}, r_{2}, \ldots, r_{m}, r_{i} \in \mathbb{R}, 0<r_{i}<a\right\}$ such that $\cup_{i=1}^{m} V\left(r_{i}, \delta_{\varepsilon}\right) \supset[0, a]$, where $\delta_{\varepsilon}=\varepsilon / 3 R$ and $V\left(r_{i}, \delta_{\varepsilon}\right)=$ $\left\{t \in J,\left|r_{i}-t\right|<\delta_{\varepsilon}\right\}$.

If there exists some $r_{j} \notin[0, a]_{\mathbb{T}}$, which means $0<r_{j}<a$ and $r_{j} \notin \mathbb{T}$, then we can find

$$
\begin{aligned}
& \alpha_{j}=\sup \left\{t \in[0, a]_{\mathbb{T}}, t<r_{j}\right\}, \\
& \beta_{j}=\inf \left\{t \in[0, a]_{\mathbb{J}}, t>r_{j}\right\} .
\end{aligned}
$$

Since $J$ is a closed subset of $\mathbb{R}$, it is clear in this case that $f_{n}\left(\alpha_{j}\right)=f_{n}\left(\beta_{j}\right)-f_{n}^{\nabla}\left(\beta_{j}\right)\left(\beta_{j}-\alpha_{j}\right)$. Then we can define

$$
f_{n}\left(r_{j}\right)=f_{n}\left(\beta_{j}\right)-f_{n}^{\nabla}\left(\beta_{j}\right)\left(\beta_{j}-r_{j}\right), \quad n \in \mathbb{N} .
$$


We claim that

$$
\left|f_{n}(t)-f_{n}\left(r_{j}\right)\right|<\frac{\varepsilon}{3} \text { holds. }
$$

In fact, there are two cases to consider.

Case $I\left(r_{j} \notin J\right)$ ). If $r_{j}<t<r_{j+1}$, then $t \geq \beta_{j}$ and by (78) we have

$$
\begin{aligned}
& \left|f_{n}(t)-f_{n}\left(r_{j}\right)\right| \\
& \quad \leq\left|f_{n}(t)-f_{n}\left(\beta_{j}\right)\right|+\left|f_{n}\left(\beta_{j}\right)-f_{n}\left(r_{j}\right)\right| \\
& \quad=\left|\int_{\beta_{j}}^{t} f_{n}^{\nabla}(s) \nabla s\right|+\left|f^{\nabla}\left(\beta_{j}\right)\left(\beta_{j}-r_{j}\right)\right| \\
& \quad \leq \int_{\beta_{j}}^{t}\left|f_{n}^{\nabla}(s)\right| \nabla s+\left|f^{\nabla}\left(\beta_{j}\right)\left(\beta_{j}-r_{j}\right)\right| \\
& \quad \leq R\left(t-\beta_{j}\right)+R\left(\beta_{j}-r_{j}\right) \leq R\left(t-r_{j}\right)<\frac{\varepsilon}{3} .
\end{aligned}
$$

If $r_{j-1}<t<r_{j}$, then $t \leq \alpha_{j}$ and by (78) we have

$$
\begin{aligned}
& \left|f_{n}(t)-f_{n}\left(r_{j}\right)\right| \\
& \leq\left|f_{n}\left(\alpha_{j}\right)-f_{n}(t)\right|+\left|f_{n}\left(r_{j}\right)-f_{n}\left(\alpha_{j}\right)\right| \\
& =\left|\int_{t}^{\alpha_{j}} f_{n}^{\nabla}(s) \nabla s\right| \\
& \quad+\mid\left(f_{n}\left(\beta_{j}\right)-f_{n}^{\nabla}\left(\beta_{j}\right)\left(\beta_{j}-r_{j}\right)\right) \\
& \quad \quad-\left(f_{n}\left(\beta_{j}\right)-f_{n}^{\nabla}\left(\beta_{j}\right)\left(\beta_{j}-\alpha_{j}\right)\right) \mid \\
& \leq \int_{t}^{\alpha_{j}}\left|f_{n}^{\nabla}(s)\right| \nabla s+\left|f^{\nabla}\left(\beta_{j}\right)\left(r_{j}-\alpha_{j}\right)\right| \\
& \leq R\left(\alpha_{j}-t\right)+R\left(r_{j}-\alpha_{j}\right) \leq R\left(r_{j}-t\right)<\frac{\varepsilon}{3} .
\end{aligned}
$$

Case II $\left(r_{j} \in J\right)$. We have in this case

$$
\left|f_{n}(t)-f_{n}\left(r_{j}\right)\right| \leq\left|\int_{t}^{\alpha_{j}} f_{n}^{\nabla}(s) \nabla s\right| \leq R\left|t-r_{j}\right|<\frac{\varepsilon}{3} .
$$

Next we will show that there exists a subsequence $\left\{f_{n^{\prime}}\right\}$ of $\left\{f_{n}\right\}$ convergent on $\Omega$. In fact, since $\left\{f_{n}\left(r_{1}\right)\right\}$ is bounded, it has a convergent subsequence $\left\{f_{n_{1}}\left(r_{1}\right)\right\}(n=1,2, \ldots)$. Similarly, $\left\{f_{n_{1}}\left(r_{2}\right)\right\}$ is bounded and therefore we can choose a convergent subsequence $\left\{f_{n_{2}}\left(r_{2}\right)\right\}(n=1,2, \ldots)$. If we repeat this process, we get

$$
\begin{aligned}
& f_{1_{1}}\left(r_{1}\right), f_{2_{1}}\left(r_{1}\right), \cdots, f_{n_{1}}\left(r_{1}\right), \cdots \\
& f_{1_{2}}\left(r_{2}\right), f_{2_{2}}\left(r_{2}\right), \cdots, f_{n_{2}}\left(r_{2}\right), \cdots \\
& f_{1_{p}}\left(r_{p}\right), f_{2_{p}}\left(r_{p}\right), \cdots, f_{n_{p}}\left(r_{p}\right), \cdots .
\end{aligned}
$$

Then $\left\{f_{n^{\prime}}(t)\right\}$ is convergent on $\Omega$ if we choose $\left\{n^{\prime}\right\}=\left\{n_{p}\right\}$. From the above argument, for any $r_{k}$, there exists a constant $N_{\varepsilon}\left(r_{k}\right)$ such that $\left|f_{\ell}\left(r_{k}\right)-f_{m}\left(r_{k}\right)\right|<\varepsilon / 3$ for $\ell, m>N_{\varepsilon}\left(r_{k}\right)$. For any fixed $t \in J$, there is some $V\left(r_{j}, \delta_{\varepsilon}\right)$ such that $t \in V\left(r_{i}, \delta_{\varepsilon}\right)$. Thus if we set

$$
N_{\varepsilon}=\max \left\{N_{\varepsilon}\left(r_{1}\right), N_{\varepsilon}\left(r_{2}\right), \ldots, N_{\varepsilon}\left(r_{p}\right)\right\},
$$

then when $\ell, .>N_{\varepsilon}, \ell, m \in\left\{n^{\prime}\right\}$, there holds

$$
\begin{aligned}
\left|f_{\ell}(t)-f_{m}(t)\right| \leq & \left|f_{\ell}(t)-f_{\ell}\left(r_{j}\right)\right|+\left|f_{\ell}\left(r_{j}\right)-f_{m}\left(r_{j}\right)\right| \\
& +\left|f_{m}\left(r_{j}\right)-f_{m}(t)\right|<\varepsilon .
\end{aligned}
$$

It therefore follows that $\left\{f_{n^{\prime}}(t)\right\}$ is convergent uniformly on $J$. Thus the proof is complete.

\section{Main Results}

Denote

$$
[g, h]=\{u(t): g(t) \leq u(t) \leq h(t), t \in J\} .
$$

In this section, we will make use of iterative technique to prove the main theorem.

Definition 21. Functions $u_{0}(t), v_{0}(t): J \rightarrow \mathbb{R}$ are said to be an upper and a lower solution of (1), respectively, if

$$
\begin{array}{r}
u_{0}^{\nabla}(t) \geq f\left(t, u_{0}(t), \int_{0}^{t} g(t, s) u_{0}(s) \nabla s\right), \\
t \in J, \quad u_{0}(0) \geq u_{0}(\rho(a)) ; \\
v_{0}^{\nabla}(t) \leq f\left(t, v_{0}(t), \int_{0}^{t} g(t, s) v_{0}(s) \nabla s\right), \\
t \in J, \quad v_{0}(0) \leq v_{0}(\rho(a)) .
\end{array}
$$

Theorem 22. Assume that $v_{0}(t) \leq u_{0}(t)$ are a lower and an upper solution of (1), respectively. Further, suppose that there exist two positive functions $p(t), q(t) \in \mathscr{R}_{v}$ such that one of the following conditions holds:

$(\mathrm{H}) \alpha=\sup _{t \in J}\{\nu(t) p(t)\}<1, \rho(a) G Q / p_{0}<$ $\min \{1,1 / M\}$

$\left(\mathrm{H}^{\prime}\right) \alpha=\sup _{t \in J}\{\nu(t) p(t)\}<1, \rho(a) G Q / p_{0}<1$ and $\rho(a)(P+\rho(a) Q G) \leq 1$.

And

$$
\begin{gathered}
f\left(t, u_{2}, v_{2}\right)-f\left(t, u_{1}, v_{1}\right) \\
\geq-p(t)\left(u_{2}-u_{1}\right)-n(t)\left(v_{2}-v_{1}\right) \quad \forall t \in J, \\
\quad v_{0}(t) \leq u_{1} \leq u_{2} \leq u_{0}(t), \quad v_{1} \leq v_{2},
\end{gathered}
$$

where $G, M$, and $p_{0}$ are defined as in Lemma 17; $P$ is as defined in Lemma 18. Then PBVP (1) has a maximal solution $u^{*}(t)$ and a minimal solution $v^{*}(t)$ in $D=\left\{u(t) \in C_{\mathrm{ld}}(J, \mathbb{R}), v_{0}(t) \leq\right.$ $\left.u(t) \leq u_{0}(t), t \in J\right\}$; moreover, there exist monotone iterative sequences $\left\{u_{n}(t)\right\}$ and $\left\{v_{n}(t)\right\}$ such that $u_{n}(t) \rightarrow$ $u^{*}(t), v_{n}(t) \rightarrow v^{*}(t)(n \rightarrow \infty)$ uniformly for $t \in J$. 
Proof. Without loss of generality, we suppose that $(\mathrm{H})$ holds. For any $h(t) \in C_{\mathrm{ld}}(J, \mathbb{R})$, we consider the following nonhomogeneous linear integrodifferential equations on time scales:

$$
\begin{gathered}
u^{\nabla}(t)=-p(t) u(t)-q(t) \int_{0}^{t} g(t, s) u(s) \nabla s+(F h)(t), \\
u(0)=u(\rho(a)),
\end{gathered}
$$

where $(F h)(t)=f(t, h(t),(G h)(t))+p(t) h(t)+q(t)(G h)(t)$. It follows by Lemma 11 that (89) has a unique solution $u_{h} \in$ $C_{\text {ld }}(J, \mathbb{R})$, and

$$
\begin{aligned}
& u_{h}(t) \\
& =\widehat{e}_{-p}(t, 0) \\
& \quad \times\left[\frac{1}{\hat{e}_{\Theta_{\gamma}(-m)}(\rho(a), 0)-1}\right. \\
& \quad \times \int_{0}^{\rho(a)} \widehat{e}_{-p}(0, \rho(s))[(F h)(s)-q(s)(G u)(s)] \nabla s \\
& \left.\quad \times \int_{0}^{t} \widehat{e}_{-p}(0, \rho(s))[(F h)(s)-q(s)(G u)(s)] \nabla s\right], \\
&
\end{aligned}
$$

We define an operator $A: C_{\mathrm{ld}}(J, \mathbb{R}) \rightarrow C_{\mathrm{ld}}(J, \mathbb{R}),(A h)(t)=$ $u_{h}(t)$ and will prove that (a) $v_{0} \leq A v_{0}(t), A u_{0} \leq u_{0}$ and (b) $A$ is increasing in $\left[v_{0}, u_{0}\right]$. To prove (a), we set $v_{1}=A v_{0}$; we have

$$
\begin{aligned}
& v_{1}^{\nabla}(t)=-p(t) v_{1}(t)-q(t)\left(G v_{1}\right)(t)+f\left(t, v_{0}(t),\left(G v_{0}\right)(t)\right) \\
&+p(t) v_{0}(t)+q(t)\left(G v_{0}\right)(t) \\
& v_{1}(0)=\left(A v_{0}\right)(0)=\left(A v_{0}\right)(\rho(a))=v_{1}(\rho(a)) .
\end{aligned}
$$

Denoting $k(t)=v_{0}-v_{1}$, then, by Definition 21 and (53), we have

$$
\begin{aligned}
& k^{\nabla}(t)= v_{0}^{\nabla}(t)-v_{1}^{\nabla}(t) \\
& \leq f\left(t, v_{0}(t),\left(G v_{0}\right)(t)\right)+p(t) v_{1}(t) \\
&+q(t)\left(G v_{1}\right)(t)-f\left(t, v_{0}(t),\left(G v_{0}\right)(t)\right) \\
&-p(t) v_{0}(t)-q(t)\left(G v_{0}\right)(t) \\
&= p(t)\left(v_{1}(t)-v_{0}(t)\right)+q(t)\left(G\left(v_{1}-v_{0}\right)(t)\right) \\
&=-p(t) k(t)-n(t)(G k)(t), \\
& k(0)=v_{0}(0)-v_{1}(0) \leq v_{0}(\rho(a))-v_{1}(\rho(a))=k(\rho(a)) .
\end{aligned}
$$

Thus by Lemma 17 we know that $k(t) \leq 0$ for all $t \in J$, which implies that $v_{0} \leq v_{1}$; that is, $v_{0} \leq A v_{0}$. Essentially, with the same method, we can show that $u_{0} \geq A u_{0}$.

To prove (b), we firstly show that $F$ is increasing. Letting $h_{1}, h_{2} \in C[J, \mathbb{R}], h_{1} \leq h_{2}$, then by (88) we have

$$
\begin{aligned}
&\left(F h_{2}\right)(t)-\left(F h_{1}\right)(t) \\
&= f\left(t, h_{2}(t),\left(G h_{2}\right)(t)\right)-f\left(t, h_{1}(t),\left(G h_{1}\right)(t)\right) \\
&+p(t)\left(h_{2}(t)-h_{1}(t)\right)+q(t)\left(G\left(h_{2}-h_{1}\right)(t)\right) \\
& \geq-p(t)\left(h_{2}(t)-h_{1}(t)\right)-n(t)\left(G\left(h_{2}-h_{1}\right)(t)\right) \\
&+p(t)\left(h_{2}(t)-h_{1}(t)\right)+q(t)\left(G\left(h_{2}-h_{1}\right)(t)\right) \\
&= 0 .
\end{aligned}
$$

Thus, $F(D)=\{F(u(t)), u(t) \in D\} \subset\left[F v_{0}, F u_{0}\right]$ is bounded. Next, we show that $A$ is increasing in $\left[v_{0}, u_{0}\right]$. Setting $u_{h_{1}}=$ $A h_{1}, u_{h_{2}}=A h_{2}$, then

$$
\begin{gathered}
u_{h_{1}}^{\nabla}(t)=-p(t) u_{h_{1}}(t)-q(t)\left(\left(G u_{h_{1}}\right)(t)\right)+F\left(h_{1}(t)\right), \\
u_{h_{1}}(0)=u_{h_{1}}(\rho(a)) ; \\
u_{h_{2}}^{\nabla}(t)=-p(t) u_{h_{2}}(t)-q(t)\left(\left(G u_{h_{2}}\right)(t)\right)+F\left(h_{2}(t)\right), \\
u_{h_{2}}(0)=u_{h_{2}}(\rho(a)) .
\end{gathered}
$$

Denote $l(t)=u_{h_{2}}(t)-u_{h_{1}}(t)$. By (88) and (93), we have

$$
\begin{gathered}
-l^{\nabla}(t) \leq-p(t)(-l(t))-q(t) \int_{0}^{t} g(t, s)(-l(s)) \nabla s, \\
-l(0)=-l(\rho(a)) .
\end{gathered}
$$

It follows by Lemma 17 that $-l(t) \leq 0, \forall t \in J$, which implies that $u_{h_{2}}(t) \geq u_{h_{1}}(t)$; that is, $A$ is increasing; thus (b) has been proved.

Let $u_{n}=A u_{n-1}$ and $v_{n}=A v_{n-1}(n=1,2, \ldots)$. By (a) and (b), we have

$$
\begin{aligned}
v_{0}(t) & \leq v_{1}(t) \leq \cdots \leq v_{n}(t) \leq \cdots \leq u_{n}(t) \\
& \leq \cdots \leq u_{2}(t) \leq u_{1}(t), \quad t \in J .
\end{aligned}
$$

Next we will show that both $\left\{u_{n}\right\}$ and $\left\{v_{n}\right\}$ have convergent subsequences. Let $U=\left\{u_{n}\right\}_{n=1}^{\infty}$. It is obvious that $U$ is a bounded set; in fact,

$$
\left|u_{n}(t)\right| \leq \max \left\{\left|v_{0}(t)\right|,\left|u_{0}(t)\right|, t \in J\right\}:=S, \quad n=1,2, \ldots
$$

From the above discussion, we have

$$
u_{n}^{\nabla}(t)=-p(t) u_{n}(t)-q(t)\left(G u_{n-1}\right)(t)+\left(F u_{n-1}\right)(t) .
$$

In view of the properties of $p, q, G$, and $F$, we know that $\left\{u_{n}^{\nabla}(t)\right\}$ is bounded on $J$; by Lemma 20 , we know that there exists a subsequence of $\left\{u_{n}\right\}$ which converges uniformly on $J$ 
to some $u^{*} \in C_{\mathrm{ld}}(J, \mathbb{R})$. Since $\left\{u_{n}^{\nabla}(t)\right\}$ is nonincreasing, we see that $\left\{u_{n}^{\nabla}(t)\right\}$ itself converges uniformly on $J$ to $u^{*} \in C_{\mathrm{ld}}(J, \mathbb{R})$. Then, by the continuity of $p, q, G$ and $F$, we have

$$
\begin{gathered}
-p(t) u_{n}(t)-q(t)\left(G u_{n-1}\right)(t)+\left(F u_{n-1}\right)(t) \\
\longrightarrow-p(t) u^{*}(t)-q(t)\left(G u^{*}\right)(t) \\
+\left(F u^{*}\right)(t) \quad \text { as } n \longrightarrow \infty .
\end{gathered}
$$

By the definition of $F$, we know that $u_{n}^{\nabla}(t)$ converges uniformly on $J$ to $f\left(t, u^{*}(t),\left(G u^{*}\right)(t)\right)$. Thus, we have

$$
\left(u^{*}\right)^{\nabla}(t)=f\left(t, u^{*}(t),\left(G u^{*}\right)(t)\right), \quad u^{*}(0)=u^{*}(\rho(a)),
$$

which implies that $u^{*}$ is solution to PBVP (1). Essentially, with the same method, we can prove that $\left\{v_{n}\right\}$ converges uniformly on $J$ to some $v^{*} \in C_{\mathrm{ld}}(J, \mathbb{R})$, and $v^{*}$ is also a solution to PBVP (1).

Finally, we try to show that $u^{*}$ and $v^{*}$ are maximal solution and minimal solution to PBVP (1), respectively, on $\left[v_{0}, u_{0}\right]$.

Suppose that $u \in C_{\mathrm{ld}}(J, \mathbb{R})$ is any solution to PBVP (1) in $\left[v_{0}, u_{0}\right]$; then there exists $k \in N$ such that $v_{k}(t) \leq u(t) \leq u_{k}(t)$. Denoting $r(t)=u-u_{k+1}$, then by (88) we have

$$
\begin{aligned}
& r^{\nabla}(t)= u^{\nabla}(t)-u_{k+1}^{\nabla}(t) \\
&= f(t, u(t),(G u)(t))+p(t) u_{k+1}(t) \\
&+q(t)\left(G u_{k+1}\right)(t)-f\left(t, u_{k}(t),\left(G u_{k}(t)\right)\right) \\
&-p(t) u_{k}(t)-q(t)\left(G u_{k}\right)(t) \\
& \leq p(t)\left(u_{k}-u\right)+q(t)\left(G\left(u_{k}-u\right)(t)\right) \\
&-p(t)\left(u_{k}-u_{k+1}\right)(t)-q(t)\left(G\left(u_{k}-u_{k+1}\right)(t)\right) \\
& \leq p(t)\left(u_{k+1}-u\right)+q(t)\left(G\left(u_{k+1}-u\right)(t)\right) \\
&=-p(t) r(t)-q(t)(G r)(t), \\
& r(0)=r(\rho(a)) .
\end{aligned}
$$

Thus, by Lemma 17, we obtain that $r(t) \leq 0$ for all $t \in J$, which implies that $u(t) \leq u_{k+1}(t)$. Similarly, we can show that $v_{k+1}(t) \leq u(t)$ for all $t \in J$. Consequently, by induction, we have $v_{n}(t) \leq u(t) \leq u_{n}(t)$ for all $t \in J, n=1,2, \ldots$. Then, by taking limits, we get $v^{*}(t) \leq u(t) \leq u^{*}(t)$ all $t \in J$, which implies that $u^{*}$ and $v^{*}$ are maximal solution and minimal solution to PBVP (1), respectively. The proof is complete.

As an application, we consider the second order PBVP on time scales:

$$
\begin{gathered}
y^{\nabla \nabla}=f\left(t, y, y^{\nabla}\right), \quad \forall t \in J ; \\
y(0)=0, \quad y^{\nabla}(0)=y^{\nabla}(\rho(a)),
\end{gathered}
$$

where $J=[0, a], f \in C[J \times \mathbb{R} \times \mathbb{R}, \mathbb{R}]$. We make the following assumptions.
$\left(\mathrm{H}_{1}\right)$ There exist $v_{0}, u_{0} \in C^{2}[J \times \mathbb{R}]$ satisfying $v_{0}^{\nabla}(t) \leq$ $u_{0}^{\nabla}(t), t \in J$ and

$$
\begin{aligned}
& v_{0}^{\nabla \nabla} \leq f\left(t, v_{0}, v_{0}^{\nabla}\right), \quad \forall t \in J ; \quad v_{0}^{\nabla}(0) \leq v_{0}^{\nabla}(\rho(a)), \\
& u_{0}^{\nabla \nabla} \geq f\left(t, v_{0}, u_{0}^{\nabla}\right), \quad \forall t \in J ; \quad u_{0}^{\nabla}(0) \geq u_{0}^{\nabla}(\rho(a)) \text {. }
\end{aligned}
$$

$\left(\mathrm{H}_{2}\right)$ There exist two positive functions $p(t), q(t)$ with $\alpha=$ $\sup \{v(t) q(t)\}<1$ such that

$$
\begin{gathered}
f\left(t, u_{2}, v_{2}\right)-f\left(t, u_{1}, v_{1}\right) \\
\geq-p(t)\left(u_{2}-u_{1}\right)-q(t)\left(v_{2}-v_{1}\right) \quad \forall t \in J, \\
v_{0}(t) \leq u_{1}(t) \leq u_{2}(t) \leq u_{0}, \\
v_{0}^{\nabla}(t) \leq u_{1}^{\nabla}(t) \leq u_{2}^{\nabla}(t) \leq u_{0}^{\nabla}(t) .
\end{gathered}
$$

Theorem 23. Suppose that conditions $\left(H_{1}\right)$ and $\left(H_{2}\right)$ are satisfied. Then there exist monotone sequences $\left\{\bar{v}_{n}\right\},\left\{\bar{u}_{n}\right\} \subset$ $C^{1}[J, \mathbb{R}]$ such that

$$
\begin{gathered}
v_{0}^{\nabla}(t)=\bar{v}_{0}(t) \leq \bar{v}_{1}(t) \leq \cdots \leq \bar{v}_{n}(t) \\
\leq \cdots \leq \bar{u}_{n}(t) \leq \cdots \leq \bar{u}_{1}(t) \leq \bar{u}_{0}(t) \\
=u_{0}^{\nabla}(t), \quad t \in J
\end{gathered}
$$

then $v^{*}(t), u^{*}(t)$ are the minimal and maximal solutions of (102) satisfying $v_{0}^{\nabla}(t) \leq v^{* \nabla}(t) \leq u^{* \nabla}(t) \leq u_{0}^{\nabla}(t)$.

Proof. Let $y^{\nabla}(t)=u(t)$. Then $y(t)=y(0)+\int_{0}^{t} u(s) \nabla s=$ $\int_{0}^{t} y(s) \nabla s$, and therefore BVP (102) reduces to the following PBVP:

$$
u^{\nabla}=f(t, G u, u), \quad \forall t \in J ; \quad u(0)=u(\rho(a)),
$$

where $(G u)(t)=\int_{0}^{t} u(s) \nabla s$. Obviously, this is a PBVP of type (1) with $g(t, s)=1$. Hence, the conclusion of Theorem 23 follows immediately from Theorem 22 . The proof is complete.

\section{Conflict of Interests}

The authors declare that there is no conflict of interests regarding the publication of this paper.

\section{Acknowledgments}

The study was supported by the Fundamental Research Funds for the Central Universities (no. 2652012141) and Beijing Higher Education Young Elite Teacher Project. 


\section{References}

[1] M. Bohner and A. Peterson, Dynamic Equations on Time Scales, An Introduction with Applications, Birkhäuser, Boston, Mass, USA, 2001.

[2] M. Bohner and A. Peterson, Advances in Dynamic Equations on Time Scales, Birkhäuser, Boston, Mass, USA, 2003.

[3] C. Corduneanu, Integral Equations and Applications, Cambridge University Press, 1991.

[4] R. Agarwal, M. Bohner, A. Domoshnitsky, and Y. Goltser, "Floquet theory and stability of nonlinear integro-differential equations," Acta Mathematica Hungarica, vol. 109, no. 4, pp. 305-330, 2005.

[5] D. Franco, "Green's functions and comparison results for impulsive integrodifferential equations," Nonlinear Analysis, vol. 47, pp. 5723-5728, 2001.

[6] D. J. Guo, "Initial value problems for integro-differential equations of Volterra type in Banach spaces," Journal of Applied Mathematics and Stochastic Analysis, vol. 7, no. 1, pp. 13-23, 1994.

[7] Z. He and X. He, "Periodic boundary value problems for first order impulsive integro-differential equations of mixed type," Journal of Mathematical Analysis and Applications, vol. 296, no. 1, pp. 8-20, 2004.

[8] J. J. Nieto and R. Rodríguez-López, "Periodic boundary value problem for non-Lipschitzian impulsive functional differential equations," Journal of Mathematical Analysis and Applications, vol. 318, no. 2, pp. 593-610, 2006.

[9] G. Song, "Initial value problems for systems of integrodifferential equations in Banach spaces," Journal of Mathematical Analysis and Applications, vol. 264, no. 1, pp. 68-75, 2001.

[10] H. Xu and J. J. Nieto, "Extremal solutions of a class of nonlinear integro-differential equations in Banach spaces," Proceedings of the American Mathematical Society, vol. 125, no. 9, pp. 26052614, 1997.

[11] Y. Xing, W. Ding, and M. Han, "Periodic boundary value problems of integro-differential equation of Volterra type on time scales," Nonlinear Analysis: Theory, Methods \& Applications, vol. 68, no. 1, pp. 127-138, 2008.

[12] D. R. Anderson, "Existence of solutions for a first-order pLaplacian BVP on time scales," Nonlinear Analysis: Theory, Methods \& Applications, vol. 69, no. 12, pp. 4521-4525, 2008.

[13] R. P. Agarwal, V. Otero-Espinar, K. Perera, and D. R. Vivero, "Multiple positive solutions of singular Dirichlet problems on time scales via variational methods," Nonlinear Analysis: Theory, Methods \& Applications, vol. 67, no. 2, pp. 368-381, 2007.

[14] C. C. Tisdell and A. Zaidi, "Basic qualitative and quantitative results for solutions to nonlinear, dynamic equations on time scales with an application to economic modelling," Nonlinear Analysis, vol. 68, no. 11, pp. 3504-3524, 2008.

[15] P. Amster, C. Rogers, and C. C. Tisdell, "Existence of solutions to boundary value problems for dynamic systems on time scales," Journal of Mathematical Analysis and Applications, vol. 308, no. 2, pp. 565-577, 2005. 


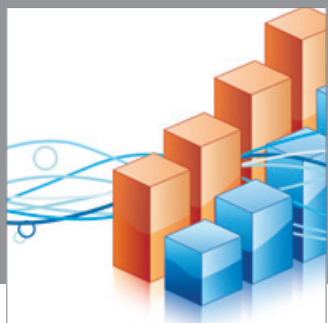

Advances in

Operations Research

mansans

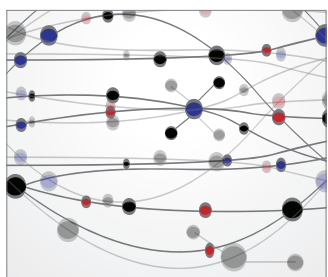

The Scientific World Journal
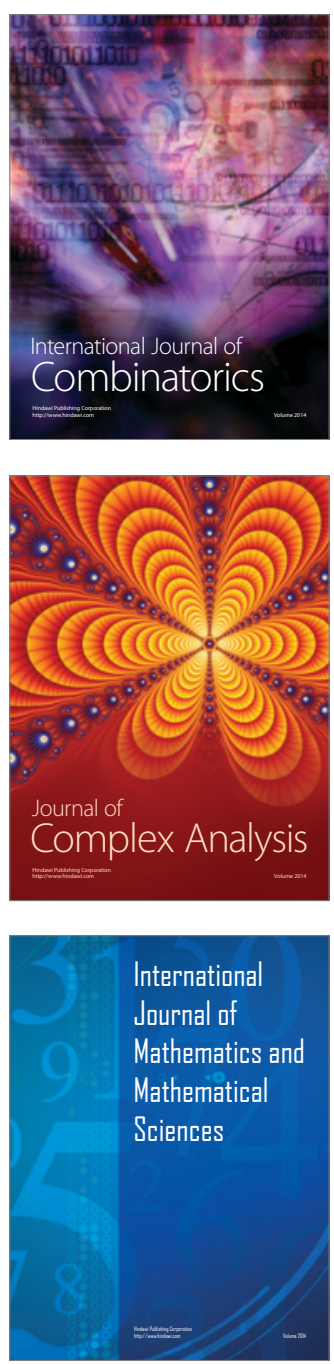
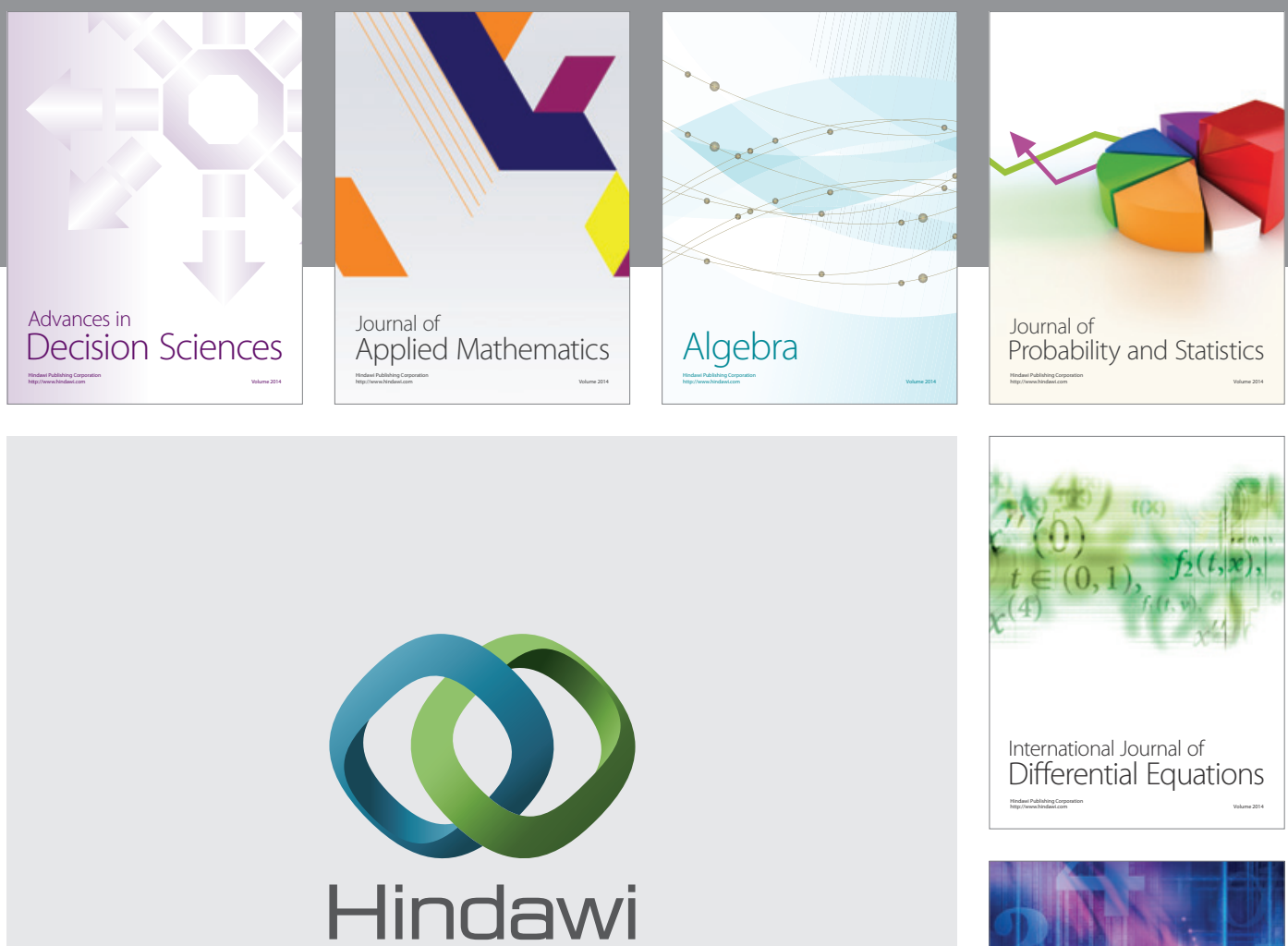

Submit your manuscripts at http://www.hindawi.com
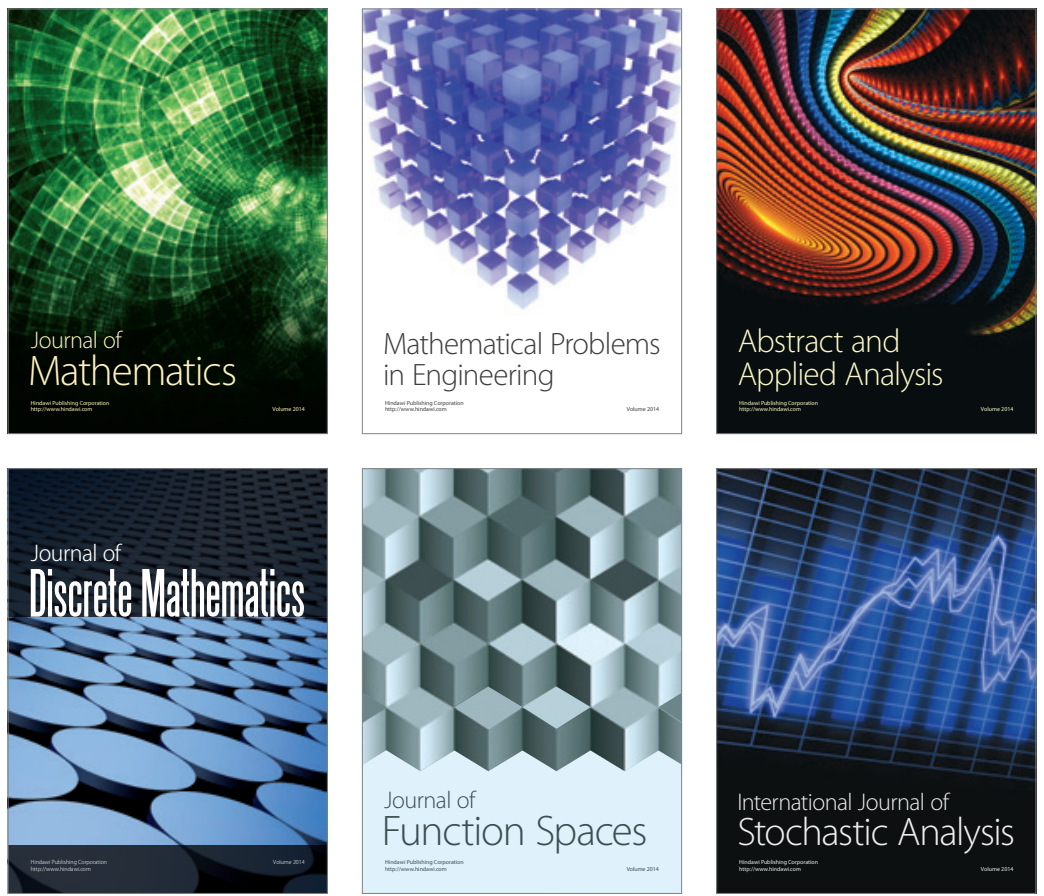

Journal of

Function Spaces

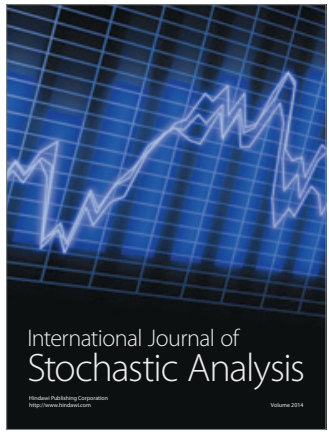

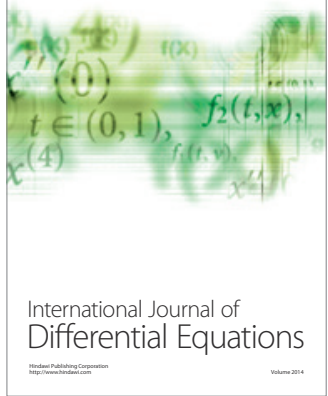
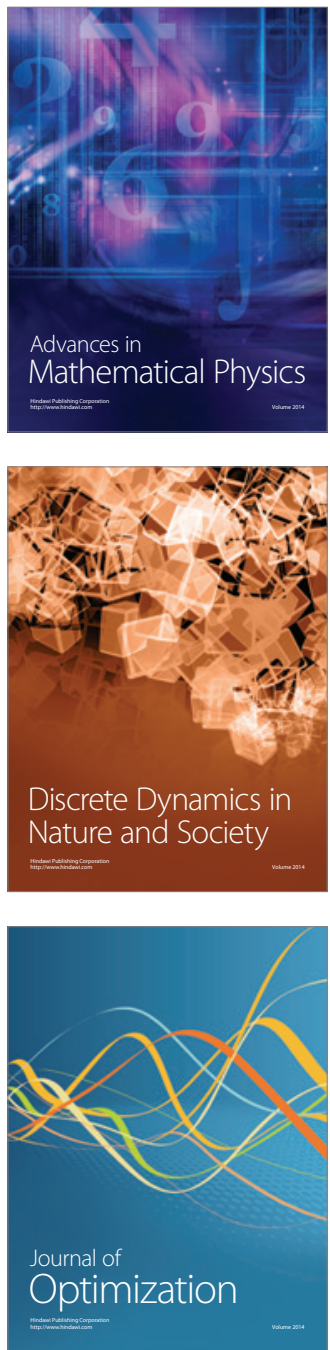